\section{Retention of item attributes in free recall*}

\author{
STEPHEN MADIGAN and LINDA DOHERTY \\ University of Southern California, Los Angeles, Calif. 90007
}

A free recall experiment using mixed-modality lists was conducted to assess retention of information about presentation format of to-be-remembered words. Lists consisted of items presented once only, auditorily or visually, or twice, either in the same modality or once in each modality. Ss were required to remember items only (standard free recall) or to remember items as well as their modality and frequency attributes. With all recall from secondary memory, there were no effects of the modality variable (auditory, visual, or mixed) on recall of repeated items, although Ss were able to retain a considerable amount of information about the modality and frequency characteristics of recalled items. Processing this additional information had the effect of lowering word recal scores relative to the standard free recall test. It is argued that recall of modality attributes is based on some direct representation of this information in memory, and is not solely the result of a "tagging" process.

The superior recall of auditorily over visually presented verbal material seems to occur in every common test of immediate recall. This modality difference also seems quite limited, extending over the last six or eight items in free recall tasks and diminishing with slower rates of presentation (Murdock \& Walker, " 1970). However, the fact that modality differences are not obtained in recall of material from long-term memory does not necessarily mean that this storage system does not preserve information about presentation modality, or that all items regardless of modality are reduced to some common format. As Underwood (1969) has suggested, it is possible to regard stimulus modality as an attribute of items in memory, which a memorizing $\mathrm{S}$ may be able to recall, in addition to recalling the to-be-remembered item itself. One of the purposes of this experiment was to assess retention of the modality attribute of items presented in mixed-modality (auditory and visual) lists, and also to assess the possible "cost" associated with requiring a $S$ to store and retrieve such information. That is, this situation might resemble the concurrent task experiment, in which item recall would decline as the additional processing task became more demanding (Posner, 1967).

A second aim of the experiment was to examine the effects of change of presentation modality on recall of repeated items. If items are presented twice in a list but their input modality changes from first to second presentation, how will recall be affected in comparison to recall of items that are presented twice in the

* This research was supported bv Grant MH 19567-01, National Institute of Mental Health. same modality? Since there seem to be no modality differences in secondary memory with single-mode lists, one might expect that modality change would be ineffective in altering recall performance. Alternatively, modality change might have a deleterious effect on recall if it promotes some failure of "trace contact" when an item is re-presented in a format different from that of its original presentation.

\section{VERBAL MATERIALS AND LISTS}

Each list consisted of 32 different words, 16 presented once and 16 presented twice, making a total of 48 presentations in a list. The twice-presented items were displayed in one of four ways: two auditory or two visual presentations (AA and VV), and mixed-modality presentations (AV or VA). Four different words were presented in each of these four ways. Of the 16 once-presented words, 8 were auditory and 8 were visual.

Each list was divided into two blocks of 24 presentations. The first block (Serial Positions 1-24) consisted of 12 auditory and 12 visual items. Four once-presented items in each modality occurred in this block; they were not subsequently re-presented. Of the remaining 8 items in each modality, 4 of each were repeated in the second block, either in the same modality ( $A A$ and VV) or in the other modality (AV and VA). Four additional items in each modality were presented for the first (and only) time in the second block (Serial Positions 25-48). The placement of items in each block was determined randomly, with the restriction that no more than 3 consecutive items could be in the same modality. The median lag (interval between the first and second presentation or repeated items) was approximately 18 items for same-mode and mixed-mode repetitions. Four such lists were prepared in two independent replications of materials. In addition, each replication was prepared with AA and VV items and positions counterbalanced and $\mathrm{AV}$ and VA items and positions counterbalanced. In all, then, there were four sets of four lists each.

\section{PROCEDURE}

Lists were presented by a closed-circuit television and videotape system. The interitem interval was 2 sec. Each $S$ was supplied with a television monitor and set of headphones. Two Ss were tested at the same time, one under standard free recall instructions and one under attribute recall instructions. In this latter condition, $\mathrm{S}$ was instructed prior to list presentation to recall items, as well as to indicate for each item to be recalled how many times it had been presented (once or twice) and its presentation modality (auditory, visual, or mixed). Ss performed this task by circling one of six alternatives (AA, AV, VA, VV, A, V) for each item recalled. (It might be noted that these attributes could not be judged completely independently of one another; e.g., Ss could not judge an item to be once-presented, mixed-mode.) Twenty-seven Ss (introductory psychology students) were tested in each of the two conditions.

In order to eliminate recency effects, an eight-digit, simultaneous auditory-visual memory span test was given immediately after presentation of the last list word. Two and one-half minutes were allowed for written recall for each of the four lists.

\section{RESULTS}

Word Recall

Probability of recall for the six types of items are shown in Table 1. The proportions for twice-presented items are based on 432 observations ( 4 items per list by 4 lists by $27 \mathrm{Ss}$ ), and the proportions for once-presented items are based on 864 observations ( 8 items per list by 4 lists by $27 \mathrm{Ss}$ ). The input position (first block and second block) had no effect on recall of once-presented items, and the data have been collapsed over this classification.

It is plain that word recall obtained under standard free recall conditions (item recall) was not at all affected by the modality manipulation; there were no significant differences among the four kinds of repeated items or between once-presented auditory and visual items. This result changes only slightly in the word recall scores for Ss recalling item attributes as well. There is a suggestion here that mixed-modality repetitions are recalled better than same-modality repetitions, but this difference, of 
Table 1

Probability of Recall of Once- and Twice-Presented Items in Item and Attribute Recall Tasks

\begin{tabular}{cccccccc} 
& \multicolumn{3}{c}{ Twice-Presented } & & \multicolumn{2}{c}{ Once-Presented } \\
\cline { 2 - 7 } Recall Task & AA & AV & VA & VV & & A & V \\
\hline $\begin{array}{c}\text { Item Recall } \\
\text { Item and } \\
\text { Attribute Recall }\end{array}$ & .41 & .42 & .41 & .43 & .17 & .20 \\
\hline
\end{tabular}

approximately $6 \%$, is only marginally $(.05<\mathrm{p}<.10)$ significant. For both sets of data, there is an obvious and unsurprising difference in recall of once- and twice-presente;items, although this comparison also suggests that the $2: 1$ ratio of recall predicted by the total-time hypothesis (Waugh, 1967) was not obtained. This might be a reflection of the inhibition of "weak" (once-presented) items by "strong" (twice-presented) items described by Tulving \& Hastie (1972).

Also apparent in Table 1 is a general lowering of word recall scores when Ss are required to process the additional information represented by the modality and frequency attributes. All six types of items reflect this "cost" in terms of number of words recalled, and the difference was significant in every case.

\section{Attribute Recall}

The distribution of attribute identifications for recalled items is shown in Table 2. The negative diagonal (underlined entries) are "correct" responses in which both the modality (auditory, visual, or mixed-mode) and frequency (once- or twice-presented) attributes were correctly specified. The rightmost column shows the recall totals on which the percentages are based.

There are a number of ways of summarizing these data, but only a few aspects of them will be discussed here. First, the main implication of these data is that Ss' judgment of item attributes contains a good deal of information about actual attributes. The largest entry in each row (i.e., for each item type) is for the correct classification. For AA, VV, A, and V items, the smallest entry in each row is that classification that cuntains neither of the attributes of the recalled item. For example, Ss never misidentified a once-presented auditory item as a repeated visual item. Ss were also able to discriminate mixed-mode from single-mode repetitions and to further discriminate between the two kinds of mixed-mode repetitions (AV and VA).

Another way of summarizing these data may be obtained by collapsing judgments for a given item type over all the attribute identifications that contain at least one mode-appropriate component. For example, for VV items, this would include VV, VA, $A V$, and $V$ identifications, which account for roughly $90 \%$ of all the responses for recalled VV items. With the exception of mixed-mode items, this method indicates that accuracy of modality judgments ranged from $86 \%$ (for $\mathrm{V}$ items) to $92 \%$ (for A items). Mixed-mode items can be collapsed over only two categories with this scoring method (i.e., AV and VA), and the accuracy estimates here are generally lower than for the other item types. Table 2 suggests that this was due to a greater tendency for mixed-mode repetitions to be identified as once-presented items.

\section{DISCUSSION}

While modality differences in secondary memory may be obtained with short (10-item) lists and fast presentation rates (Murdock \& Walker, 1970), the present results clearly indicate that presentation modality and consistency of presentation modality are irrelevant variables as far as item recall is concerned. At the same time, attribute identifications were anything but random, indicating some extensive representation of modality-specific information in memory. It is possible, of course, to ascribe attribute judgments to a "tagging" of items at input. The fact that there was a significant cost associated with attribute recall might support this suggestion. An $S$ attempting to remember information about item characteristics simply has less time to devote to rehearsing and organizing the to-be-remembered words. That is, $\mathbf{S}$ could presumably recall any discriminable feature of input, as long as he deliberately encoded and stored this information. There are, however, a number of reasons for thinking that item attributes are, in fact, represented more directly in memory-that the "trace" somehow directly reflects stimulus modality (Murdock, 1967). One is simply that the cost observed in this experiment might, in part, reflect output interference; Ss were making a six-alternative forced choice for each item recalled. When $S s$ are required to recall attributes in a way that does not produce output interference-when the attribute task is introduced after word presentation and recall, for example-one still finds considerable accuracy in recall of some kinds of attributes (Hintzman \& Block, 1970; Zimmerman \& Underwood, 1968). A second argument against the "tagging" notion is that the cost that was obtained here seems relatively slight, at least in respect to the amount of additional modality and frequency information that was being recalled. When Ss are required to remember which of two digits (a " 1 " or a " 2 ") accompanied each word in a list, word recall losses may approach $50 \%$ in comparison to free recall conditions, and recall of such an arbitrary "attribute" may itself be quite poor (Madigan, 1969). Average word recall losses in the present study were less than $10 \%$, and accuracy was quite high for some combinations of the two attributes.

Finally, changing an item's presentation format-the voice in which it is spoken-from an initial to a second (test) presentation adversely affects recognition of that item at the second presentation (Kirsner \& Craik, 1971). This indirectly indicates preservation of an item attribute. It also suggests that in the present study, there should have been more recognition failures for second presentations of mixed-mode items. No direct measure of this was taken, however, and any possible failures of such "trace contact" had no effects on recall in any event.

\section{REFERENCES}

HINTZMAN, D., \& BLOCH, R. Repetition and memory: Evidence for a mutiple trace hypothesis. Journal of Experimental Psychology, 1971, 88, 297-306.

KIRSNER, K., \& CRAIK, F. I. M. Naming and decision processes in short-term recognition memory. Journal of Experimental Psychology, 1971, 88, 149-157.

MADIGAN, S. Effects of processing additional binary information in free

Table 2

Distribution of Attribute Identifications (Percent)

\begin{tabular}{|c|c|c|c|c|c|c|c|}
\hline \multirow{2}{*}{$\begin{array}{l}\text { Item } \\
\text { Type }\end{array}$} & \multicolumn{6}{|c|}{ Attribute Identification } & \multirow{2}{*}{$\begin{array}{l}\text { Recall } \\
\text { Totals }\end{array}$} \\
\hline & AA & AV & $\mathrm{VA}_{\mathrm{A}}$ & $\mathrm{VV}$ & A & $\mathrm{V}$ & \\
\hline $\mathbf{A A}$ & 39.5 & 16.5 & 20.1 & 5.7 & 14.3 & 3.6 & 139 \\
\hline AV & $\overline{6.5}$ & 50.0 & 19.4 & 5.2 & 8.4 & 10.3 & 154 \\
\hline VA & 5.9 & $\overline{20.9}$ & 46.7 & 7.7 & 10.1 & 8.3 & 167 \\
\hline VV & 7.5 & 12.1 & $\overline{14.4}$ & 41.6 & 3.0 & 21.2 & 132 \\
\hline A & 12.0 & 16.6 & 4.6 & $\overline{0.0}$ & 58.3 & 8.3 & 108 \\
\hline V & 2. 3 & 8.6 & 8.6 & 3.1 & 11.7 & 65.6 & 128 \\
\hline
\end{tabular}


recall. Paper presented at the meetings of the Western Psvchological Association. Los Angeles, April 1970.

MURDOCK, B. B., JR. Auditory and visual stores in short-term memory. Act sy'chologica, 1967, 27, 316-324.

MURDOCK, B. B. JR.. \& WALKER, K. D.

Modality effects in free recall. Journal of

Verbai Learning \& Verbal Behavior.
$1970.8 .665-676$

POSNER, M. I, Short-term memory systems in human information processing. Acta Psychologica, 1967, 27, 267-284.

TULVING, E., \& HASTIE. R. Inhibition effects of intralist repetition in free recall. Journal of Experimental Psycholozy. 1972 , in press.

UNDERWOOD, B. J. Attributes of memory.
Psychological Review, 1969, 76, 559-573

WAUGH, N. C. Presentation time and free recall. Journal of Experimental Psy chology, 1967, 73, 39-44.

ZIMMERMAN, J., \& UNDERWOOD, B. J. Ordinal position knowledge within and across lists as a function of instructions in free-recall learning. Journal of General Psychology, 1968, 79,301-307. 